\title{
FREQÜÊNCIA DOS EFEITOS DO ÁLCOOL NO FETO E PADRÃO DE CONSUMO DE BEBIDAS ALCOÓLICAS PELAS GESTANTES DE MATERNIDADE PÚBLICA DA CIDADE DE SÃO PAULO
}

\author{
FREQUENCY OF ALCOHOL EFFECTS IN FETUS AND PATTERN OF \\ ALCOHOL CONSUMPTION BY PREGNANT WOMEN AT A PUBLIC \\ MATERNITY HOSPITAL IN SÃO PAULO CITY, BRAZIL
}

Maria dos Anjos Mesquita ${ }^{1}$

Conceição Aparecida de Mattos Segre ${ }^{2}$

Mesquita MA, Segre CAM. Freqüência dos efeitos do álcool no feto e padrão de consumo de bebidas alcoólicas pelas gestantes de maternidade pública da cidade de São Paulo. Rev Bras Crescimento Desenvolvimento Hum. 2009; 19(1): 63-77

\section{Resumo:}

Objetivo: avaliar a frequência dos efeitos do álcool no feto e o padrão de consumo de bebidas alcoólicas pelas gestantes de maternidade pública da cidade de São Paulo. Método: foram examinados 1964 recém-nascidos vivos, e entrevistadas as suas mães por meio de perguntas diretas e pelo questionário T-ACE. Quantificou-se o consumo semanal de álcool pelas puérperas, três meses antes e durante a gestação e compararam-se as características físicas dos recém-nascidos com esse padrão de consumo. Foram utilizados os testes de Mann-Whitney, teste qui-quadrado e o coeficiente de correlação de Spearman. Adotou-se o nível de significância de 5\%. Resultados: Em 76 crianças (38,69/1000 nascidos vivos) identificou-se o espectro de desordens fetais alcoólicas. Três delas (1,52/1000 nascidos vivos) tinham síndrome alcoólica fetal. Três meses antes da gravidez, 43,90\% das mulheres consumiram álcool semanalmente. Na gravidez esse número caiu para 21,20\% no primeiro trimestre, $17,50 \%$ no segundo e $17,10 \%$ no terceiro e $33,29 \%$ das puérperas consumiram álcool em algum momento da gestação. O questionário T-ACE foi positivo em 31,11\% puérperas e negativo em $68,84 \%$. Quanto maior a quantidade de álcool consumido, durante os primeiros seis meses gestacionais, menor o peso de nascimento, perímetro cefálico e comprimento dos recém-nascidos. Essas medidas não apresentaram diferenças significativas entre as puérperas T-ACE positivas e negativas. Conclusões: Foram encontrados 76 recém-nascidos com o espectro de desordens fetais alcoólicas. O uso de álcool pelas gestantes foi alto, sugerindo a realização de rastreamento rotineiro desse consumo.

Palavras-chave: Recém-nascidos; gestantes; álcool; síndrome alcoólica fetal; transtornos relacionados ao uso de álcool.

1 Médica Pediatra e Neonatologista Hospital Municipal Maternidade-Escola de Vila Nova Cachoeirinha "Dr. Mário de Moraes Altenfelder Silva” e Hospital e Maternidade Cruz Azul. Mestre

2 Livre-Docente. Orientadora do programa de pós-graduação em Ciências da Saúde do Instituto de Assistência ao Servidor Público Estadual

Local do trabalho- Hospital Municipal Maternidade-Escola de Vila Nova Cachoeirinha "Dr. Mário de Moraes Altenfelder Silva”, São Paulo, Brasil

Correspondência para: manjosmesquita@uol.com.br 


\begin{abstract}
:
Objetive: to evaluate the frequency of alcohol effects in fetus and the pattern of alcohol consumption by pregnant women of a public maternity hospital in the city of São Paulo, Brazil. Method: one thousand sixty four live newborns were evaluated and their mothers interviewed by direct questions and by T-ACE questionnaire. The weekly intake of alcohol by pregnant women was quantified in the previous three months and during the gestation, and the physical characteristics of newborns compared with the consumption pattern. The MannWhitney test, Chi-square test and Spearman rank correlation coefficient were used. The significant level of 5\% was adopted. Results: in 76 children (38.69/1000 live births) the fetal alcohol spectrum disorders was identified. Three children (1.52/1000 live births) presented fetal alcohol syndrome. In the previous three months of pregnancy, 43.90\% pregnant women consumed alcohol weekly. During the gestation the numbers decreased to $21.20 \%$ in first trimester, $17.50 \%$ in the second and $17.10 \%$ in the third and $33.29 \%$ of pregnant women reported drinking alcohol at some time during pregnancy. The T-ACE questionnaire was positive in $31.11 \%$ pregnant women and negative in $68.84 \%$. The amount of alcohol consumption during the first six months of gestation was related to lower the weight, head circumference and length of newborns. These measures did not present significant differences between pregnant women T-ACE positive and negative. Conclusion: seventy six newborns with fetal alcohol spectrum disorders were found. The frequency of alcohol use by pregnant women was high and suggests the routine follow-up of the alcohol consume during pregnancy.
\end{abstract}

Key words: newborn; pregnant women; alcohol; fetal alcohol syndrome; alcohol-related disorders

\section{INTRODUÇÃO}

A exposição intra-uterina ao álcool, resultante do consumo de bebidas alcoólicas pela gestante, leva a vários efeitos deletérios ao embrião e ao feto. Esses, agrupados sobre o termo espectro desordens fetais alcoólicas (fetal alcohol spectrum disorders - FASD) incluem alterações físicas, mentais, comportamentais e/ou de aprendizado. Existem grandes chances de essas alterações se perpetuarem por toda a vida com alto risco de os indivíduos afetados se tornarem dependentes de álcool e de outras drogas, terem problemas mentais, dificuldades escolares e no trabalho, comportamento sexual inapropriado e problemas com a justiça. ${ }^{1,7}$

O FASD engloba a síndrome alcoólica fetal (SAF), os defeitos congênitos relacionados ao álcool (alcohol-related birth defects -
ARBD) e as desordens de neurodesenvolvimento relacionadas ao álcool (alcohol-related neurodevelopmental disorders - ARND), sendo o quadro mais grave representado pela primeira. ${ }^{1,3}$

Crianças com SAF têm alterações faciais características, restrição de crescimento pré e/ ou pós-natal e anormalidades estruturais e/ou funcionais do sistema nervoso central (SNC). Podem apresentar outras malformações congênitas, dificuldades de aprendizado, de memória, problemas funcionais, emocionais e de comportamento. ${ }^{1,8}$

Os efeitos nocivos do álcool, na prole de mães alcoólatras, parece ser o problema mais trágico do alcoolismo. ${ }^{9} \mathrm{O}$ FASD leva a prejuízos para a criança afetada, para a sua família e para toda a comunidade. ${ }^{4}$ Atualmente, considera-se que o álcool é o agente teratogênico fetal mais comum, tendo-se tornado um problema de saúde pública. ${ }^{9}$ 
As anomalias congênitas presentes no FASD são totalmente preveníveis se a mulher se abstiver de ingerir álcool imediatamente antes da concepção e durante a gravidez. ${ }^{2,8,10 \text {, }}$ ${ }^{12}$ Assim elas necessitam ser rotineiramente rastreadas quanto ao uso de álcool durante o pré-natal. 5, 10, 11, 13, 15

Apesar da clínica pela exposição prénatal ao álcool ser bem conhecida, existe grande dificuldade no diagnóstico do FASD. ${ }^{1,3} \mathrm{Pe}-$ las suas conseqüências, os efeitos do álcool nos filhos das mães alcoólatras devem ser precocemente identificados. Necessitam ter um seguimento adequado e multiprofissional de todos os seus problemas garantindo-lhes melhor adequação familiar e social. ${ }^{1,10}$

Embora o consumo de álcool pelas gestantes possa levar a consequências devastadoras e permanentes ao seu concepto, elas não despertam a merecida importância nos profissionais de saúde nem nos órgãos do governo brasileiro.

O objetivo é verificar a frequência dos efeitos do álcool no feto das gestantes usuárias de bebidas alcoólicas em um hospital público terciário da cidade de São Paulo, avaliando o seu padrão de consumo, a associação entre o uso de álcool com o de outros fatores pelas grávidas e comparar as variáveis dos recém-nascidos (RN) de gestantes usuárias ou não de álcool.

\section{MÉTODO}

O trabalho constituiu-se em um estudo observacional transversal analítico de RN nascidos vivos e de suas respectivas mães, realizado no Hospital Municipal Maternidade-Escola de Vila Nova Cachoeirinha "Dr. Mário de Moraes Altenfelder Silva” (HMEVNC), hospital público terciário da cidade de São Paulo, de 13/08/2006 a 21/01/2008.

Nesse período nasceram vivas no hospital 7447 RN e nele adentraram, após parto não hospitalar, 33 crianças. Foram incluídos os RN admitidos entre a $0 \mathrm{~h} 1 \mathrm{~min}$ de domingo e às $23 \mathrm{~h}$ 59min de segunda-feira durante o período citado. Esse período foi escolhido por motivos de ordem operacionais ligados á disponibilidade de uma das pesquisadoras (MAM). Excluídos os RN que apresentavam alguma síndrome genética, foram a óbito, transferidos para outro hospital ou receberam alta antes do exame físico e/ou da entrevista materna, a mãe foi a óbito antes da entrevista, não eram o primeiro gemelar e eram filhos de puérperas que se recusaram a participar da pesquisa.

As variáveis coletadas dos $\mathrm{RN}$ foram idade gestacional (IG), características do filtro nasal e do nariz e as medidas antropométricas da fenda palpebral (FP), lábio superior (LS), peso de nascimento (PN), perímetro cefálico (PC) e comprimento (C).

A FP foi medida com os olhos do paciente fechados pela dificuldade das crianças dessa faixa etária em abri-los. A presença ou ausência de nariz antevertido e do filtro nasal liso foram analisadas subjetivamente pela pesquisadora. O PC, a FP e a maior largura do LS foram realizados entre $24-72 \mathrm{~h}$ de vida. O RN estava em decúbito dorsal, em estado de sono ou alerta passivo. Para análise das medidas do PN, PC, C, FP e da maior largura do LS foram elaboradas curvas de percentil, baseadas nas medidas das crianças deste estudo e as medidas foram consideradas anormais se menores ou iguais ao $10^{\circ}$ percentil para a IG.

Ultra-som transfontanelar foi realizado na presença de alterações anatômicas do crânio e/ou quando o PC era menor ou igual ao $10^{\circ}$ percentil, pela curva de percentil de Usher utilizada como guia, nos RN de mães consumidoras de álcool. ${ }^{16}$ Ultra-som abdominal e RX dos ossos e de tórax foram realizados nos que apresentavam alterações propedêuticas torácicas, abdominais, urogenitais e nos membros. Avaliação pelo cardiologista e pelo neurologista foi feita na presença de alterações cardíacas e neurológicas nos RN. 
Posteriormente ao exame físico de cada $\mathrm{RN}$, foi preenchido um formulário mediante respostas obtidas, por entrevista estruturada com questões fechadas, das respectivas mães. Coletadas das puérperas a idade em anos completos, coabitação com o companheiro, remuneração mensal, anos completos de escolaridade, planejamento da gravidez atual, número e época de início das consultas do pré-natal e uso de bebidas alcoólicas pelos que coabitavam com ela. Em relação a cada trimestre da atual gestação e aos últimos três meses anteriores à gravidez foi coletado o uso de drogas lícitas e ilícitas e a quantidade de álcool consumida semanalmente por cada puérpera.

A quantidade de bebida alcoólica consumida foi transformada em unidades de consumo de álcool. Para se verificar a existência de diferenças em relação à confirmação ou não do consumo de álcool por cada puérpera, primeiro foram feitas perguntas diretas e logo em seguida foi aplicado o questionário T-ACE padronizado por Sokol et al. ${ }^{17}$

Essas mulheres foram divididas em TACE positivo e negativo dependendo da pontuação final do seu questionário, em consumidoras se referiam consumo de álcool e em não consumidoras se não o consumiam.

Baseado nos critérios do Centers for Disease Control and Prevention (CDC) americano de 2004, considerou-se como tendo SAF os RN com relato materno de consumo de álcool durante a sua vida intra-uterina, as três alterações faciais características (FP pequena, filtro nasal liso, LS fino), restrição de crescimento (PN e/ou PC e/ou $\mathrm{C}<10^{\circ}$ percentil) e alterações estruturais e/ou neurológicas do SNC. ${ }^{3}$

Segundo os critérios do U.S. Institute of Medicine (IOM) de 1996, elucidados por Hoyme et al., foram considerados como tendo ARBD os RN com relato materno de consumo de álcool durante a gravidez, pelo menos duas alterações faciais típicas e uma ou mais malformações congênitas e como tendo ARND aqueles com relato materno de consumo de álcool durante a gestação e alterações estruturais e/ou neurológicas do SNC. ${ }^{2}$

Alterações estruturais do SNC corresponderam ao PC menor ou igual ao $10^{\circ}$ percentil e/ou a alteração ultrassonográfica da imagem cerebral. Convulsão, tremor, irritabilidade, alteração de sucção/deglutição, não relacionadas a outras causas, foram consideradas alterações neurológicas. Os outros critérios neurológicos recomendados pelo roteiro de CDC foram desconsiderados neste estudo pela dificuldade em serem avaliados no RN.

As características físicas dos neonatos foram comparadas em relação à presença ou não do consumo de álcool pelas suas mães e também com o padrão do seu uso.

A coleta de dados, o exame físico do RN e a entrevista materna foram realizados por uma das pesquisadoras (MAM).

Os testes estatísticos usados, de acordo com as variáveis estudadas, foram o coeficiente de correlação de Spearman $\left(\mathrm{r}_{\mathrm{s}}\right)$, teste de Mann-Whitney (Z) e qui-quadrado $\left(c^{2}\right)$. Adotou-se o nível de significância de 5\%.

A participação das puérperas e dos seus filhos foi consentida após a leitura e assinatura pelas primeiras do Termo de Consentimento de Livre Esclarecimento dos Pais ou Responsável Legal elaborado para fins específicos deste estudo.

Este estudo foi aprovado pelo Comitê de Ética em Pesquisa do HMEVNC pelo protocolo 009/2006.

\section{RESULTADOS}

No período do estudo nasceram 7480 crianças vivas sendo 7447 de parto hospitalar e 33 de parto não hospitalar. Pelo fator de inclusão, 2035 RN poderiam participar da pesquisa. Foram excluídas seis $(0,29 \%)$ por serem sindrômicas, 11 (0,54\%) por terem ido a óbito antes do exame físico, sete $(0,34 \%)$ por terem 
recebido alta antes da análise física e da entrevista materna, uma $(0,05 \%)$ por ter sido transferida para outro hospital antes do exame físico, uma (0,05\%) pela morte da sua mãe antes da entrevista e nove $(0,44 \%)$ porque as suas mães se recusaram a participar. Das 2000 $(98,28 \%)$ crianças que restaram, 36 eram de gestações bigemelares e o segundo gêmeo foi desconsiderado. A amostra para análise ficou constituída de 1964 RN (96,51\%) e de suas respectivas mães.

As puérperas tinham uma idade média de 25,06 anos, variando de 13 a 44 anos, desvio padrão de 6,54. Co-habitavam com o companheiro 1516 (77,20\%).

A quantidade de salários mínimos mensais das puérperas foi de zero a cinco, com média de 0,73 e desvio padrão de 1,13.

As puérperas estudaram em média 8,38 anos, variando entre nenhum a 17 anos, com desvio padrão de 2,83.

A gravidez não foi planejada por 1402 (71,40\%) dessas mulheres.

Sessenta (3,05\%) puérperas não tiveram em nenhuma consulta médica durante a gestação e nove $(0,45 \%)$ não souberam informar quantas consultas fizeram. Das restantes, a mediana de consultas foi de seis, o número máximo de 20, desvio padrão de 2,81. A média do início do pré-natal ocorreu no $3^{\circ}$ mês de gestação (2,72 $\pm 1,55$ meses)

O padrão de consumo, de bebidas alcoólicas, uso de cigarro e de drogas ilícitas pelas puérperas nos quatro períodos estudados está descrito na tabela 1. Do total das puérperas, 654 (33,29\%) consumiram álcool em algum momento da gravidez sendo que dessas, $140(21,41 \%)$ consumiram-no nos seus três trimestres. O número de puérperas consumidoras de álcool e as unidades totais por elas consumidas semanalmente diminuíram com o progredir da gestação. Responderam positivamente ao questionário T-ACE $611(31,11 \%)$ das puérperas e negativamente 1352 (68,84\%). Uma $(0,05 \%)$ não soube responder às suas questões. Das T-ACE negativas, 188 (13,90\%) consumiram alguma quantidade de álcool durante a gravidez.

A mediana de coabitantes das puérperas usuários de álcool foi de uma pessoa, variando de 0 a 4, com desvio padrão de 0,67.

Nas tabelas 2 e 3 podem ser vistas as correlações entre o consumo de álcool e as variáveis das puérperas com os diversos testes estatísticos e o nível de significância p.

As puérperas que consumiram álcool nos três meses antes da gestação e no primeiro trimestre foram significativamente mais novas que as não consumidoras ( $\mathrm{Z}: \mathrm{p}=0,001 /$ prégestação e $p=0,014 / 1^{\circ}$ trimestre). A quantidade de unidades de álcool consumida também foi maior entre as puérperas mais jovens nos dois períodos citados $\left(\mathrm{r}_{\mathrm{s}}: \mathrm{p}=0,002 /\right.$ pré-gestação e $\mathrm{p}=0,014 / 1^{\circ}$ trimestre). Com a progressão da gravidez essas diferenças desapareceram.

Quanto à remuneração, não foram encontradas diferenças significativas entre as puérperas que consumiram ou não álcool na gestação e nos três meses que a antecederam (Z: p $=0,209 /$ pré-gestação, $\mathrm{p}=0,083 / 1^{\circ}$ trimestre, $\mathrm{p}=0,204 / 2^{\circ}$ trimestre e $\mathrm{p}=0,358 / 3^{\circ}$ trimestre).

Nos três meses pré-gestacionais e no primeiro trimestre de gestação não houve diferença significativa da escolaridade entre as consumidoras ou não de álcool. As puérperas que continuaram a consumir álcool no segundo e no terceiro trimestres tinham menor escolaridade $\left(\mathrm{Z}: \mathrm{p}=0,018 / 2^{\circ}\right.$ trimestre e $\mathrm{p}=0,000 / 3^{\circ}$ trimestre).

As puérperas que consumiram álcool tiveram o início do pré-natal mais tardio, quando testadas em toda a gestação ( $\mathrm{Z}: \mathrm{p}=0,000 / 1^{\circ}$ trimestre, $\mathrm{p}=0,019 / 2^{\circ}$ trimestre e $\mathrm{p}=0,020 / 3^{\circ}$ trimestre). Quanto maior a quantidade de álcool consumido semanalmente, mais tardio foi o começo do pré-natal $\left(\mathrm{r}_{\mathrm{s}}: \mathrm{p}=0,000 / 1^{\circ}\right.$ trimestre, $\mathrm{p}=0,018 / 2^{\circ}$ trimestre e $\mathrm{p}=0,031 / 3^{\circ}$ trimestre).

As consumidoras de álcool durante a gravidez fizeram um número menor de con- 
sultas durante o pré-natal ( $\mathrm{Z}: \mathrm{p}=0,000 / 1^{\circ}$ trimestre, $\mathrm{p}=0,003 / 2^{\circ}$ trimestre e $\mathrm{p}=0,010 / 3^{\circ}$ trimestre). Quanto maior a quantidade de álcool consumida por semana, menor o número de consultas realizadas $\left(\mathrm{r}_{\mathrm{s}}: \mathrm{p}=0,000 / 1^{\circ}\right.$ trimestre, $\mathrm{p}=0,001 / 2^{\circ}$ trimestre e $\mathrm{p}=0,005 /$ $3^{\circ}$ trimestre).

O número de coabitantes das puérperas que consumiram álcool foi maior entre aque- las que também o consumiram (Z: $\mathrm{p}=0,000$ nos quatro períodos estudados).

As puérperas que planejaram a gestação foram menos consumidoras de álcool $\left(\chi^{2}: \mathrm{p}=0,017 /\right.$ pré-gestação, $\mathrm{p}=0,000 / 1^{\circ}$ trimestre, $\mathrm{p}=0,005 / 2^{\circ}$ trimestre e $\mathrm{p}=0,023 / 3^{\circ}$ trimestre) e consumiram significativamente menor quantidade nos quatro períodos estuda$\operatorname{dos}\left(\mathrm{Z}: \mathrm{p}=0,018 /\right.$ pré-gestação, $\mathrm{p}=0,000 / 1^{\circ}$

Tabela 1: Padrão de consumo de bebidas alcoólicas, cigarro e de drogas ilícitas pelas puérperas antes e nos três trimestres de gravidez

\begin{tabular}{|c|c|c|c|c|c|}
\hline Variáveis & & 3 meses Pré-G & $1 \mathrm{~T}$ & $2 \mathrm{~T}$ & $3 \mathbf{T}$ \\
\hline \multicolumn{6}{|l|}{ UTAC/semana } \\
\hline Mínimo & & 0,00 & 0,00 & 0,00 & 0,00 \\
\hline Máximo & & 192,50 & 174.22 & 192,50 & 192,50 \\
\hline Média & & 2,25 & 0,93 & 0,81 & 0,74 \\
\hline Desvio padrão & & 10,47 & 6,68 & 7,87 & 8,22 \\
\hline Consumidoras semanais de álcool & & 3 meses Pré-G & $1 \mathrm{~T}$ & $2 \mathrm{~T}$ & $3 \mathrm{~T}$ \\
\hline \multirow[t]{2}{*}{ Não } & $\mathrm{N}$ & 1102 & 1548 & 1620 & 1628 \\
\hline & $\%$ & 56,10 & 78,80 & 82,50 & 82,90 \\
\hline \multirow[t]{2}{*}{ Sim } & $\mathrm{N}$ & 862 & 416 & 344 & 336 \\
\hline & $\%$ & 43,90 & 21,20 & 17,50 & 17,10 \\
\hline \multirow{3}{*}{$\begin{array}{c}\text { Consumidoras diárias de cigarro } \\
\text { Não }\end{array}$} & & 3 meses Pré-G & $1 \mathrm{~T}$ & $2 \mathrm{~T}$ & $3 \mathbf{T}$ \\
\hline & $\mathrm{N}$ & 1355 & 1490 & 1576 & 1640 \\
\hline & $\%$ & 69,00 & 75,90 & 80,20 & 81,70 \\
\hline Sim & $\mathrm{N}$ & 609 & 474 & 388 & 360 \\
\hline & $\%$ & 31,00 & 24,10 & 19,80 & 18,30 \\
\hline \multirow{3}{*}{$\begin{array}{c}\text { Consumidoras de drogas ilícitas } \\
\text { Não }\end{array}$} & & 3 meses Pré-G & $1 \mathrm{~T}$ & $2 \mathrm{~T}$ & $3 \mathbf{T}$ \\
\hline & $\mathrm{N}$ & 1922 & 1943 & 1951 & 1951 \\
\hline & $\%$ & 97,90 & 98,90 & 99,30 & 99,30 \\
\hline \multirow[t]{2}{*}{ Sim } & $\mathrm{N}$ & 42 & 21 & 13 & 13 \\
\hline & $\%$ & 2,10 & 1,10 & 0,70 & 0,70 \\
\hline
\end{tabular}

Pré-G: pré-gestação; 1 T: primeiro trimestre; 2 T: segundo trimestre; 3 T: terceiro trimestre; UTAC: unidades totais de álcool consumidas 
Tabela 2: Correlações entre o consumo de bebidas alcoólicas e as variáveis das puérperas

\begin{tabular}{|c|c|c|c|c|c|c|c|c|}
\hline \multirow[b]{2}{*}{ Variáveis das puérperas } & \multicolumn{2}{|c|}{3 meses Pré-G } & \multicolumn{2}{|c|}{$1 \mathrm{~T}$} & \multicolumn{2}{|c|}{$2 \mathrm{~T}$} & \multicolumn{2}{|c|}{$3 T$} \\
\hline & \multicolumn{6}{|c|}{ Consumo semanal de álcool } & & \\
\hline Idade (anos) & Não & Sim & Não & Sim & Não & Sim & Não & Sim \\
\hline Média & 25,49 & 24,51 & 25,22 & 24,43 & 25,11 & 24,80 & 25,06 & 25,03 \\
\hline $\mathbf{Z}$ & \multicolumn{2}{|c|}{3,408} & \multicolumn{2}{|c|}{2,468} & \multicolumn{2}{|c|}{0,598} & \multicolumn{2}{|c|}{0,102} \\
\hline $\mathbf{p}$ & \multicolumn{2}{|c|}{$0,001(*)$} & \multicolumn{2}{|c|}{$0,014(*)$} & \multicolumn{2}{|c|}{0,550} & \multicolumn{2}{|c|}{0,919} \\
\hline UTAC & \multicolumn{2}{|c|}{$-0,070$} & \multicolumn{2}{|c|}{$-0,055$} & \multicolumn{2}{|c|}{$-0,008$} & \multicolumn{2}{|c|}{0,003} \\
\hline $\mathbf{r}_{\mathrm{s}}$ & \multicolumn{2}{|c|}{$0,002(*)$} & \multicolumn{2}{|c|}{$0,014(*)$} & \multicolumn{2}{|c|}{0,728} & \multicolumn{2}{|c|}{0,897} \\
\hline Remuneração (SM) & Não & Sim & Não & Sim & Não & Sim & Não & Sim \\
\hline Média & 0,76 & 0,70 & 0,75 & 0,66 & 0,75 & 0,67 & 0,74 & 0,68 \\
\hline $\mathbf{Z}$ & \multicolumn{2}{|c|}{1,255} & \multicolumn{2}{|c|}{1,734} & \multicolumn{2}{|c|}{1,269} & \multicolumn{2}{|c|}{0,918} \\
\hline $\mathbf{p}$ & \multicolumn{2}{|c|}{0,209} & & 83 & & 04 & & \\
\hline Escolaridade (anos de estudo) & Não & Sim & Não & Sim & Não & Sim & Não & Sim \\
\hline Média & 8,33 & 8,46 & 8,42 & 8,28 & 8,44 & 8,13 & 8,48 & 7,94 \\
\hline Zp & 0,0150 & 987 & $1,6930,0$ & $2,3650,01$ & $(*) 3,69$ & $000(*)$ & & \\
\hline Coab usuários de álcool & Não & Sim & Não & Sim & Não & Sim & Não & Sim \\
\hline Média & 0,55 & 0,88 & 0,64 & 0,91 & 0,65 & 0,90 & 0,65 & 0,89 \\
\hline $\mathbf{Z}$ & & 539 & & & & & & \\
\hline $\mathbf{p}$ & & $00(*)$ & & $(*)$ & & $0(*)$ & 0,0 & $(*)$ \\
\hline Início do pré-natal (mês) & Não & Sim & Não & Sim & Não & Sim & Não & Sim \\
\hline Média & & & 2,64 & 3,03 & 2,68 & 2,93 & 2,69 & 2,87 \\
\hline $\mathbf{Z}$ & & & & 705 & & & & \\
\hline $\mathbf{p}$ & & & & $(*)$ & & $(*)$ & & $(*)$ \\
\hline UTAC & & & & & & & & \\
\hline $\mathbf{r}_{\mathrm{s}}$ & & & & & & & & 49 \\
\hline $\mathbf{p}$ & & & 0,0 & & & $(*)$ & & $1(*)$ \\
\hline Consultas no pré-natal & Não & Sim & Não & Sim & Não & Sim & Não & Sim \\
\hline Média & & & 6,63 & 6,03 & 6,60 & 6,05 & 6,59 & 6,11 \\
\hline $\mathbf{Z}$ & & & & & & & & \\
\hline $\mathbf{p}$ & & & & $(*)$ & & $(*)$ & 0,0 & $(*)$ \\
\hline UTAC & & & & & & & & \\
\hline $\mathbf{r}$ & & & & & & & & \\
\hline $\mathbf{p}$ & & & & $(*)$ & & $(*)$ & 0,0 & $(*)$ \\
\hline
\end{tabular}

Pré-G: pré-gestação; 1 T: primeiro trimestre; 2 T: segundo trimestre; 3 T: terceiro trimestre; SM: salário mínimo; Z: teste de Mann-Whitney; $r_{s}$ :coeficiente de correlação de Spearman; p: nível de significância; $(*)$ : significativo; UTAC: unidades totais de álcool consumidas; coab: coabitantes 
Tabela 3: Variáveis das puérperas, correlacionadas como o consumo de bebidas alcoólicas

\begin{tabular}{|c|c|c|c|c|c|c|c|c|c|c|c|c|}
\hline & \multicolumn{3}{|c|}{3 meses Pré-G1 } & \multicolumn{3}{|c|}{$\mathbf{T}$} & \multicolumn{3}{|c|}{$2 \mathrm{~T}$} & \multicolumn{3}{|c|}{$3 \mathrm{~T}$} \\
\hline & & \multicolumn{11}{|c|}{ Consumo semanal de álcool } \\
\hline $\begin{array}{l}\text { Variáveis das } \\
\text { puérperas }\end{array}$ & $\begin{array}{c}\text { Não } \\
\text { N }\end{array}$ & $\underset{\mathbf{N}}{\operatorname{Sim}}$ & Média & $\begin{array}{c}\text { Não } \\
\text { N }\end{array}$ & $\underset{\mathbf{N}}{\operatorname{Sim}}$ & Média & $\begin{array}{c}\text { Não } \\
\text { N }\end{array}$ & $\underset{\mathbf{N}}{\operatorname{Sim}}$ & Média & $\begin{array}{c}\text { Não } \\
\text { N }\end{array}$ & $\underset{\mathbf{N}}{\operatorname{Sim}}$ & Média \\
\hline \multicolumn{13}{|l|}{$\begin{array}{l}\text { Planejamento } \\
\text { da gravidez }\end{array}$} \\
\hline Não & 63 & 639 & 2,52 & 1076 & 326 & 1,14 & 1135 & 267 & 0,96 & 1145 & 257 & 0,84 \\
\hline Sim & 339 & 223 & 1,57 & 472 & 90 & 0,39 & 485 & 77 & 0,45 & 483 & 79 & 0,50 \\
\hline$\chi^{2}$ & & 5,667 & & & 12,59 & & & 7,928 & & & 5,168 & \\
\hline $\mathbf{p}$ & & $0,017(*)$ & & & $0,000(*)$ & & & $0,005(*)$ & & & $0,023(*)$ & \\
\hline $\mathbf{Z}$ & & 2,372 & & & 3,497 & & & 2,902 & & & 2,235 & \\
\hline $\mathbf{p}$ & & $0,018(*)$ & & & $0.000\left(^{*}\right)$ & & & $0,004(*)$ & & & $0,025(*)$ & \\
\hline \multirow{2}{*}{\multicolumn{13}{|c|}{$\begin{array}{l}\text { Coabitação com } \\
\text { o companheiro }\end{array}$}} \\
\hline & & & & & & & & & & & & \\
\hline Não & 219 & 229 & 3,54 & 323 & 125 & 2,03 & 344 & 104 & 1,35 & 363 & 85 & 1,05 \\
\hline Sim & 883 & 633 & 1,87 & 1225 & 291 & 0,60 & 1276 & 240 & 0,65 & 1265 & 251 & 0,65 \\
\hline$\chi^{2}$ & & 12,30 & & & 15,702 & & & 13,048 & & & 1,424 & \\
\hline p & & $50,000(*)$ & & & $0,000(*)$ & & & $0,000(*)$ & & & 0,233 & \\
\hline $\mathbf{Z}$ & & 4,725 & & & 4,252 & & & 3,841 & & & 1,371 & \\
\hline $\mathbf{p}$ & & $0,000(*)$ & & & $0,000(*)$ & & & $0,000(*)$ & & & 0,170 & \\
\hline \multicolumn{13}{|l|}{$\begin{array}{l}\text { Consumo de } \\
\text { cigarros }\end{array}$} \\
\hline Não & 897 & 458 & & 1276 & 214 & & 1368 & 208 & & 1405 & 199 & \\
\hline Sim & 205 & 404 & & 272 & 202 & & 252 & 136 & & 223 & 137 & \\
\hline$\chi^{2}$ & \multicolumn{3}{|c|}{180,62} & \multicolumn{3}{|c|}{171,94} & \multicolumn{3}{|c|}{102,92} & \multicolumn{2}{|c|}{136,40} & \\
\hline p & \multicolumn{3}{|c|}{$0,000(*)$} & \multicolumn{3}{|c|}{$0,000(*)$} & \multicolumn{3}{|c|}{$0,000(*)$} & \multicolumn{2}{|c|}{$0,000(*)$} & \\
\hline \multicolumn{13}{|l|}{$\begin{array}{l}\text { Consumo de } \\
\text { drogas ilícitas }\end{array}$} \\
\hline Não & 1102 & 820 & & 1540 & 403 & & 1615 & 336 & & 1622 & 329 & \\
\hline Sim & 0 & 42 & & 8 & 13 & & 5 & 8 & & 6 & 7 & \\
\hline$\chi^{2}$ & \multicolumn{3}{|c|}{54,87} & \multicolumn{3}{|c|}{21,09} & \multicolumn{2}{|c|}{17,55} & & \multicolumn{2}{|c|}{12,45} & \\
\hline p & \multicolumn{3}{|c|}{$0,000(*)$} & \multicolumn{2}{|c|}{$0,000\left(^{*}\right)$} & & \multicolumn{2}{|c|}{$0,000\left(^{*}\right)$} & & \multicolumn{2}{|c|}{$0,000(*)$} & \\
\hline
\end{tabular}

Pré-G: pré-gestação; 1 T: primeiro trimestre; 2 T: segundo trimestre; 3 T: terceiro trimestre; $c^{2}$ : teste qui-quadrado; Z: teste de Mann-Whitney; p: nível de significância; $\left({ }^{*}\right)$ : significativo

trimestre, $\mathrm{p}=0,004 / 2^{\circ}$ trimestre e $\mathrm{p}=0,025 / 3^{\circ}$ trimestre).

Houve associação entre o consumo de álcool e de cigarros pelas puérperas tanto nos três meses que antecederam a gravidez quanto nos seus três trimestres $\left(\chi^{2}: p=0,000\right)$. O mesmo aconteceu entre consumo de álcool e de drogas ilícitas pelas puérperas nos quatro períodos estudados $\left(\chi^{2}: \mathrm{p}=0,000\right)$.

As puérperas que coabitaram com o companheiro foram menos consumidoras de álcool $\left(\boldsymbol{\chi}^{2}: \mathrm{p}=0,000\right)$ e consumiram menor quantidade por semana $(Z: p=0,000)$ antes da gravidez e nos dois primeiros trimestres da gravidez. No último trimestre da gestação essa diferença não foi significativa.

A média da IG das crianças deste estudo foi de 38,55 semanas, variando de 25 a 43 , com desvio padrão de 2,49. O PN médio foi de $3048,51 \mathrm{~g}$, variando de $540 \mathrm{~g}$ a $5680 \mathrm{~g}$, com desvio padrão de $613,32 \mathrm{~g}$. O PC médio foi de $34,34 \mathrm{~cm}$, variando de $20,00 \mathrm{~cm}$ a $38,50 \mathrm{~cm}$, com desvio padrão de $2,02 \mathrm{~cm}$. O C médio foi de $47,80 \mathrm{~cm}$, variando de $28,00 \mathrm{~cm}$ a $56,00 \mathrm{~cm}$, com desvio padrão de $3,16 \mathrm{~cm}$. A medida média da FP foi de 
$1,97 \mathrm{~cm}$, variando de $0,80 \mathrm{~cm}$ a $3,00 \mathrm{~cm}$, com desvio padrão de $0,24 \mathrm{~cm}$. O LS teve uma medida média de $0,51 \mathrm{~cm}$, variando de $0,20 \mathrm{~cm}$ a $0,90 \mathrm{~cm}$, com desvio padrão de 0,11.

O filtro nasal foi normal em 1842 (93,79\%) RN e liso em 121 (6,16\%). O lábio leporino em uma criança impediu a caracterização do seu filtro. Nariz antevertido esteve presente em $279(14,20 \%)$ RN e ausente em 1685 (85,80\%).

Os RN foram classificados em pequenos, adequados e grandes para a IG segundo as curvas de percentil elaboradas a partir da população estudada.

Na tabela 4 correlacionam-se as unidades de álcool consumidas pelas puérperas com as variáveis do RN. Quanto maior a quantidade de álcool consumido semanalmente pelas puérperas, durante os dois primeiros trimestres da gestação, menor o PN $\left(r_{s}: p=0,003 / 1^{\circ}\right.$ trimestre e $\mathrm{p}=0,001 / 2^{\circ}$ trimestre), o PC $\left(r_{s}: p=0,015 / 1^{\circ}\right.$ trimestre e $\mathrm{p}=0,016 /$ $2^{\circ}$ trimestre) e o $\mathrm{C}\left(\mathrm{r}_{\mathrm{s}}: \mathrm{p}=0,033 / 1^{\circ}\right.$ trimestre e $\mathrm{p}=0,002 / 2^{\circ}$ trimestre) dos RN. Essa correlação não é significativa em relação ao período pré-gestacional e ao terceiro trimestre de gestação. A quantidade de consumo de álcool pelas puérperas não ter qualquer associação com na IG e nas medidas da FP e do LS dos RN.

A tabela 5 mostra a relação entre as variáveis do RN com as puérperas T-ACE positivas e negativas. Nenhuma das medidas antropométricas do RN apresentou diferenças

Tabela 4: Correlações das unidades de álcool consumidas semanalmente pelas puérperas com as variáveis dos recém-nascidos.

\begin{tabular}{|c|c|c|c|c|c|}
\hline & \multirow{2}{*}{$\begin{array}{l}\text { Coeficiente de } \\
\text { correlação de } \\
\text { Spearman }\left(r_{s}\right)\end{array}$} & \multicolumn{4}{|c|}{ Unidades totais de álcool consumidas/semana } \\
\hline & & 3 meses Pré-G & $1 \mathrm{~T}$ & $2 \mathrm{~T}$ & $3 \mathbf{T}$ \\
\hline \multirow[t]{3}{*}{ IG } & $\mathbf{r}_{\mathrm{s}}$ & 0,019 & $-0,007$ & $-0,022$ & 0,014 \\
\hline & $\mathbf{p}$ & 0,404 & 0,755 & 0,320 & 0,537 \\
\hline & $\mathbf{N}$ & 1964 & 1964 & 1964 & 1964 \\
\hline \multirow[t]{3}{*}{ PN (g) } & $\mathbf{r}_{\mathrm{s}}$ & $-0,043$ & $-0,068$ & $-0,073$ & $-0,014$ \\
\hline & $\mathbf{p}$ & 0,055 & $0,003(*)$ & $0,001(*)$ & 0,527 \\
\hline & $\mathbf{N}$ & 1964 & 1964 & 1964 & 1964 \\
\hline \multirow[t]{3}{*}{ PC (cm) } & $\mathbf{r}_{\mathrm{s}}$ & $-0,031$ & $-0,055$ & $-0,054$ & $-0,039$ \\
\hline & $\mathbf{p}$ & 0,172 & $0,015(*)$ & $0,016(*)$ & 0,085 \\
\hline & $\mathbf{N}$ & 1964 & 1964 & 1964 & 1964 \\
\hline \multirow[t]{3}{*}{$C(\mathbf{c m})$} & $\mathbf{r}_{\mathrm{s}}$ & $-0,033$ & $-0,048$ & $-0,071$ & $-0,010$ \\
\hline & $\mathbf{p}$ & 0,143 & $0,033(*)$ & $0,002(*)$ & 0,669 \\
\hline & $\mathbf{N}$ & 1964 & 1964 & 1964 & 1964 \\
\hline \multirow[t]{3}{*}{$\mathrm{FP}(\mathrm{cm})$} & $\mathbf{r}_{\mathrm{s}}$ & $-0,015$ & $-0,014$ & 0,010 & 0,014 \\
\hline & $\mathbf{p}$ & 0,516 & 0,547 & 0,674 & 0,538 \\
\hline & $\mathbf{N}$ & 1964 & 1964 & 1964 & 1964 \\
\hline \multirow[t]{3}{*}{ LS (cm) } & $\mathbf{r}_{\mathrm{s}}$ & 0,003 & $-0,012$ & $-0,025$ & $-0,019$ \\
\hline & $\mathbf{p}$ & 0,911 & 0,589 & 0,269 & 0,402 \\
\hline & $\mathbf{N}$ & 1964 & 1964 & 1964 & 1964 \\
\hline
\end{tabular}

Pré-G: pré-gestação; 1 T: primeiro trimestre; 2 T: segundo trimestre; 3 T: terceiro tri- mestre; IG: idade gestacional; PN: peso de nascimento; PC: perímetro cefálico; C: com-primento; FP: fenda palpebral; LS: lábio superior; p: nível de significância; $\left({ }^{*}\right)$ : significativo 
Tabela 5: Média, teste de Mann-Whitney e nível de significância p entre as variáveis dos recémnascidos e o T-ACE das puérperas.

\begin{tabular}{|c|c|c|c|c|c|}
\hline Variáveis de $\mathbf{R N}$ & T-ACE & $\mathbf{N}$ & Média & $\begin{array}{c}\text { Teste de } \\
\text { Mann-Whitney } \\
\text { (Z) } \\
\end{array}$ & $\mathbf{P}$ \\
\hline \multirow[t]{2}{*}{ IG } & negativo & 1352 & 38,51 & 0,198 & 0,843 \\
\hline & positivo & 611 & 38,65 & & \\
\hline \multirow[t]{2}{*}{ PN (g) } & negativo & 1352 & 3049,67 & 0,788 & 0,430 \\
\hline & positivo & 611 & 3045,07 & & \\
\hline \multirow[t]{2}{*}{ PC $(\mathbf{c m})$} & negativo & 1352 & 34,34 & 1,082 & 0,279 \\
\hline & positivo & 611 & 34,33 & & \\
\hline \multirow[t]{2}{*}{$\mathrm{C}(\mathrm{cm})$} & negativo & 1352 & 47,80 & 0,792 & 0,428 \\
\hline & positivo & 611 & 47,79 & & \\
\hline \multirow[t]{2}{*}{$\mathbf{F P}(\mathrm{cm})$} & negativo & 1352 & 1,97 & 0,721 & 0,471 \\
\hline & positivo & 611 & 1,98 & & \\
\hline \multirow[t]{2}{*}{ LS (cm) } & negativo & 1352 & 0,25 & 0,267 & 0,790 \\
\hline & positivo & 611 & 0,23 & & \\
\hline
\end{tabular}

Pré-G: pré-gestação; 1 T: primeiro trimestre; 2 T: segundo trimestre; 3 T: terceiro trimestre; IG: idade gestacional; PN: peso de nascimento; PC: perímetro cefálico; C: comprimento; FP: fenda palpebral; LS: lábio superior; p: nível de significância

significativas entre o grupo das T-ACE positivas e negativas.

O teste qui-quadrado e o seu nível de significância $\mathrm{p}=0,024$ mostraram maior associação entre a presença de nariz antevertido no RN e a positividade do T-ACE das puérperas. $\mathrm{O}$ mesmo não ocorreu entre a presença de filtro liso e a positividade do T-ACE ( $\mathrm{c}^{2}$ : $\mathrm{p}=0,275$ ).

O cruzamento dos resultados das variáveis dos RN com o das puérperas deste estudo permitiu o achado do FASD em 76 crianças (38,69/1000 nascidos vivos). Destas, em três RN foi feito o diagnóstico de SAF (1,52/1000 nascidos vivos) em seis, o de ARBD seria possível (3,05/1000 nascidos vivos) e 67 teriam chance de ter ARND (34,11/1000 nascidos vivos).

\section{DISCUSSÃO}

A ação do álcool sobre o embrião e o feto pode causar efeitos irreversíveis com graves conseqüências individuais e sociais..$^{1,7}$

O diagnóstico do RN com FASD é difícil por nem todas as características típicas se- rem aparentes nessa idade. Muitos casos são perdidos pelo desconhecimento do padrão de consumo de álcool pela mãe, pela pouca experiência médica sobre essa doença e pela dificuldade em se avaliar o neurodesenvolvimento, as funções cognitivas e o comportamento dos pacientes nessa faixa etária., ${ }^{8}$ Mesmo quando se conhece o padrão de consumo de bebidas alcoólicas pela mãe muitos casos não são diagnosticados. $^{18}$

A prevalência média mundial da SAF é de $0,5-2$ casos por 1000 nascidos vivos. ${ }^{3,7}$ Entre as crianças participantes do presente estudo, a prevalência do diagnóstico de SAF foi compatível com a estimada pelo CDC. No entanto, a provável prevalência da forma incompleta da síndrome, representada pela ARBD e ARND, foi maior que a esperada. Segundo a U.S. Surgeon General 's Advisory estima-se que para cada criança com SAF existam três que não apresentam todas as suas características. ${ }^{12}$

O diagnóstico de SAF é mais fácil dos dois aos onze anos, uma vez que as dismorfias faciais tornam-se mais evidentes e a disfunção típica do SNC emerge clinicamente. ${ }^{5,7,9,10,19} \mathrm{~A}$ prevalência das doenças relacionadas ao uso 
de álcool na gravidez, baseada na monitoração das características físicas dos $\mathrm{RN}$, pode ser subestimada pois as suas características não estão totalmente definidas. ${ }^{7}{ }^{19}$ Assim, seria necessário o seguimento das crianças deste estudo para que melhor definição da prevalência do FASD fosse feita.

Dados publicados em 2007 pelo I Levantamento Nacional sobre os Padrões de Consumo de Álcool na População Brasileira, mostram que $32 \%$ das mulheres com $14-17$ anos e 41\% com mais de 18 anos bebem pelo menos uma vez ao ano. ${ }^{20}$ As puérperas do presente estudo, antes de engravidarem, apresentaram um consumo superior ao da média da população feminina nacional talvez pela diversidade da metodologia empregada.

Segundo Passini Júnior, 20\% das mulheres brasileiras consomem álcool na gestação. ${ }^{21}$ Trabalho de Moraes e Reichenheim mostra que 40,6\% das puérperas consumiram bebidas alcoólicas em algum momento da gestação. ${ }^{13}$ No presente estudo, o número de puérperas consumidoras de álcool durante a gravidez situouse entre os valores dos estudos referidos anteriormente.

Estudo de May et al., entre puérperas da África do Sul, e de Ethen et al., entre puérperas americanas, mostraram uma diminuição gradativa do número de gestantes consumidoras de bebidas alcoólicas com o avançar da gestação. ${ }^{22,23} \mathrm{O}$ mesmo foi encontrado no presente trabalho.

A informação correta da quantidade de bebidas alcoólicas consumida durante a gravidez é difícil de ser obtida. ${ }^{24}$ Admitir que consumiram álcool durante a gravidez pode parecer vergonhoso para as puérperas, principalmente se continuarem a consumi-lo. ${ }^{1 \text {, }}$ ${ }^{3}$ Segundo Sokol et al., o questionário T-ACE, em relação à anamnese convencional, é mais eficiente para identificar gestantes usuárias de álcool. ${ }^{17}$

No presente estudo praticamente não houve diferença em relação à freqüência do consumo de bebidas alcoólicas pelas puérperas e o modo de questionamento. Quando ele foi direto, a percentagem de puérperas consumidoras foi discretamente maior que a positividade do questionário T-ACE. Como as duas formas de questionamento foram feitas às mesmas mulheres, na mesma entrevista e referentes ao mesmo período, esse resultado não confirmou a superioridade do questionário T-ACE, para a detecção do uso de álcool pelas gestantes, ao contrário do relatado por Sokol et al. ${ }^{17}$

A negatividade do questionário TACE, neste estudo, não implicou na abstinência do uso de álcool na gravidez conforme relato de uso de bebidas alcoólicas durante a gestação por algumas puérperas T-ACE negativas. Assim, o T-ACE negativo não garantiu que o embrião e o feto estivessem livres dos efeitos da exposição ao álcool. A determinação da quantidade de álcool lesivo ao desenvolvimento embriológico e fetal é complexa e dependente de fatores que influenciam o pico da alcoolemia materna após o seu consumo. ${ }^{15}$ Uma vez que não foi determinada a quantidade segura de álcool que pode ser usada pela gestante, recomenda-se que elas se abstenham de beber. ${ }^{1,3,6,11,12,15}$

As unidades totais de álcool consumidas semanalmente pelas puérperas deste estudo diminuíram com o progredir da gravidez. Esse dado é compatível com o descrito por Ethen et al. que mostraram uma diminuição da freqüência das puérperas beberem em "binge” de 8,1\% no primeiro trimestre de gravidez para $0,5 \%$ no terceiro. ${ }^{23}$

Três meses antes da gravidez e no primeiro trimestre de gestação as puérperas que consumiram álcool eram mais jovens que as não consumidoras. Em relação à quantidade de unidades de álcool consumidas, no mesmo período, as mães mais jovens consumiram maior quantidade. Nos seis últimos meses de gestação o consumo ou não de álcool e a quantidade 
consumida não dependeu da idade. O mesmo estudo de Ehhen et al., citado anteriormente, constatou que a percentagem de grávidas que bebem aumenta com a idade e que beber em "binge" é mais comum nas mais jovens. ${ }^{23}$

Diversos estudos mostram maiores consumo de bebidas alcoólicas entre as gestantes que não vivem com o companheiro. ${ }^{13,20,25} \mathrm{O}$ mesmo foi observado no presente estudo.

Neste estudo a baixa renda das puérperas, em média menor que um salário mínimo mensal, não mostrou nenhuma influência no seu consumo de bebidas alcoólicas. Não foi um fator contribuinte para a ingestão de álcool, ao contrário do relatado pela National Institute on Alcohol Abuse and Alcoholism e pelo CDC..$^{3,7}$ A ausência dessa influência pode demonstrar que as puérperas continuam gastando os seus escassos recursos em álcool, desviando-os de outras prioridades.

Quanto à escolaridade, antes da gravidez e no primeiro trimestre, os anos de estudo das puérperas consumidoras ou não de álcool deste estudo foi semelhante. Já nos dois últimos trimestres da gravidez as que continuaram a consumir álcool tinham menor escolaridade. A baixa escolaridade constitui um fator de risco para o uso de álcool pelas gestantes segundo alguns autores. ${ }^{13,22}$ Para outros, o número de grávidas que bebem álcool aumenta proporcionalmente ao aumento da escolaridade e rendimento familiar. ${ }^{23}$

Alguns estudos relataram fatores de risco associados às gestantes consumidoras pesadas de álcool e relacionados à SAF, ARBD e ARND. ${ }^{3,7,13,22}$ Vários fatores contribuintes estiveram presentes neste estudo. Entre eles a falta de planejamento da gravidez, a associação do uso de álcool com outras drogas lícitas e ilícitas, o número de coabitantes também usuários de etanol e a não coabitação com o companheiro.

Metade das gestações na Austrália e Estados Unidos não são planejadas. ${ }^{8} 14$ Embora esse número seja muito alto, ele foi bem menor em relação à população de puérperas deste estudo.

A constatação de que o planejamento da gravidez associou-se a um consumo significativamente menor da quantidade de álcool no período pré-conceptual e durante a gestação foi comprovada no presente trabalho.

A média do mês de início do pré-natal e do número de consultas realizadas durante a gestação das puérperas desta pesquisa esta de acordo com o preconizado pelo Ministério da Saúde do Brasil. ${ }^{26}$ Apesar disso, nem todas as puérperas diminuíram ou pararam de consumir bebidas alcoólicas durante a gestação, talvez pelo fato de não terem sido alertadas sobre os perigos da ação do álcool no seu concepto. Há que se notar que, quanto maior a quantidade de unidades de álcool consumidas semanalmente pelas puérperas deste estudo, mais tardio foi o início do pré-natal e menor o seu número consultas.

No presente estudo, a coabitação com pessoas que consumiram bebidas alcoólicas, nos quatro períodos estudados, foi maior entre as puérperas que também as consumiram. A associação entre o consumo de álcool com o hábito de fumar e de usar drogas ilícitas pelas puérperas, antes e durante a gravidez, também se mostrou altamente significativa.

No trabalho elaborado por Freire et al. todos os RN das puérperas T-ACE positivas tiveram parâmetros antropométricos, em relação ao PN, PC e C, reduzidos quando comparados aos das mães T-ACE negativas, apesar de só o C apresentar redução significativa. ${ }^{25}$ No presente estudo essa correlação não foi vista nem para as medidas citadas nem para as medidas da FP e do diâmetro do LS. No entanto, quando se considerou a quantidade de unidades de álcool consumida semanalmente pelas puérperas durante os dois primeiros trimestres de gestação, observou-se que quanto maior o consumo de bebidas alcoólicas, menor o PN, o PC e o C dos RN. A inexistência da mesma correlação no terceiro 
trimestre de gestação com essas medidas talvez se deva ao fato de que o efeito nocivo do etanol seja mais intenso nas primeiras fases da gravidez conforme citado por diversos autores. ${ }^{10,12,15}$ Assim, no presente estudo, a detecção dos efeitos do álcool sobre o PN, PC e $\mathrm{C}$ ao nascimento foi maior quando se considerou a quantidade de unidades de etanol consumidas pelas grávidas do que quando se considerou o questionário T-ACE.

Apesar de subjetiva, a presença de nariz antevertido nas crianças do presente estudo mostrou-se estar associada à positividade do T-ACE das puérperas.

A identificação do FASD no RN permitirá um seguimento precoce e adequado. $\mathrm{O}$ alto consumo de álcool pelas gestantes obriga o seu rastreamento durante o pré-natal.

Um aspecto limitante do presente estudo refere-se ao fato de as questões relativas ao consumo de álcool e das outras drogas não terem sido intercaladas com outras questões que tratassem de outros tipos de comportamentos e hábitos alimentares das puérperas. Desse modo mecanismos de alerta talvez tenham sido despertados nessas mulheres levando-as a diminuir ou até a omitir o uso de bebidas alcoólicas.

Outro aspecto a ser assinalado está no fato de a classificação do PC inicial dos RN ter sido feita pela curva de percentil de Usher, elaborada para crianças com características diferentes das do presente trabalho. Por esse motivo, em algumas crianças com PC menor ou igual ao $10^{\circ}$ percentil, segundo a curva de percentil resultante das medidas das crianças do presente estudo, não foi realizado o ultrasom transfontanelar.
De acordo com os resultados obtidos neste estudo pode-se concluir que na população de RN estudada foram reconhecidos três casos de síndrome alcoólica fetal, seis teriam grande possibilidade de ter os defeitos congênitos relacionados ao álcool e em 67 as desordens de neurodesenvolvimento relacionadas ao álcool seriam possíveis.

Antes da gravidez o consumo de álcool entre as mães desta população foi alto (43,90\%) diminuindo durante a gestação. As perguntas diretas detectaram 33,29\% de puérperas consumidoras de álcool durante a gravidez e o questionário T-ACE identificou 31,11\%. Das puérperas usuárias de bebidas alcoólicas, 21,41\% delas consumiram álcool nos três trimestres da gestação.

As unidades de álcool consumidas diminuíram com o progredir da gestação. A gravidez não planejada, a não coabitação com o companheiro, o uso de outras drogas lícitas e ilícitas e a coabitação com outros consumidores de álcool mostraram associação positiva com o consumo de álcool pelas puérperas. A quantidade de unidades de álcool consumida semanalmente pelas puérperas associou-se negativamente na época de inicio do pré-natal e no número de consultas;

O número de unidades de álcool consumido pelas mães mostrou associação negativa com o peso de nascimento, perímetro cefálico e comprimento dos recém-nascidos. O questionário T-ACE não mostrou diferenças estatísticas entre as medidas antropométricas dos recém-nascidos e as mães T-ACE positivas ou negativas.

As autoras declaram não haverem conflitos de interesses. 


\section{REFERÊNCIAS}

1. Bertrand J, Floyd RL, Weber MK. Guidelines for identifying and referring persons with fetal alcohol syndrome. MMWR Recomm Rep. 2005;54(RR11):1-12.

2. Hoyme HE, May PA, Kalberg WO, Kodituwakku P, Gossage JP, Trujillo $\mathrm{PM}$, et al. A practical clinical approach to diagnosis of fetal alcohol spectrum disorders: clarification of the 1996 Institute of Medicine criteria. Pediatrics. 2005;115(1):39-47.

3. Fetal alcohol syndrome: guidelines for referral and diagnosis. National Center on Birth Defects and Developmental Disabilities, Centers for Disease Control and Prevention, Department of Health and Human Services in coordination with National Task Force on Fetal Alcohol Syndrome and Fetal Alcohol Effect [Internet]. 2004 Jul [cited 2008 Mar 8]. Available from:http:// www.cdc.gov/ncbddd/fas/documents/ FAS_guidelines_accessible.pdf.

4. Chudley AE, Conry J, Cook JL, Loock C, Rosales T, LeBlanc N. Fetal alcohol spectrum disorder: Canadian guidelines for diagnosis. CMAJ. 2005;172(5 Suppl):1S-21S.

5. Prenatal exposure to alcohol. Alcohol Res Health. 2000;24(1):32-41.

6. What is fetal alcohol syndrome?

National Organization on Fetal Alcohol Syndrome [Internet]. 2003 [cited 2003 Jun 15]. Available from:http:// www.nofas.org/main/what_is_FAS.htm.

7. May PA, Gossage JP. Estimating the prevalence of fetal alcohol syndrome: a summary. National Institute on Alcohol

Abuse and Alcoholism (NIAAA)

[Internet]. 2001 [cited 2003 Jul 18].

Available from:http://

www.niaaa.nih.gov/publications/ marh25-3/159-167.htm.

8. Floyd RL, O`Connor MJ, Sokol RJ, Bertrand J, Cordero JF. Recognition and prevention of fetal alcohol syndrome. Obstet Gynecol. 2005;106(5 Pt 1):1059-64.

9. Thackray H, Tifft C. Fetal alcohol syndrome. Pediatr Rev. 2001; 22(2):47-55.

10. Jones MW, Bass WT. Fetal alcohol syndrome. Neonatal Nets. 2003; 22(3):63-70.

11. Jacobs EA, Copperman SM, Joffe A, Kulig J, McDonald CA, Rogers PD et al. Fetal alcohol syndrome and alcoholrelated neurodevelopmental disorders. Pediatrics. 2000;106(2):358-61.

12. U.S. Surgeon General Advisory on alcohol use in pregnancy. Washington, DC:US Department of Health and Human Services [Internet]. 2005 Feb [cited 2008 Apr 2]. Available from: http:/ /www.surgeongeneral.gov/pressreleases/ sg02222005.html.

13. Moraes CL, Reichenheim ME. Rastreamento de uso de álcool por gestantes de serviços públicos de saúde do Rio de Janeiro. Rev Saúde Pública. 2007;41(5):695-703.

14. Peadon E, O‘Leary C, Bower C, Elliott E. Impacts of alcohol use in pregnancy the role of the GP. Aust Family Physician. 2007;36(11):935-39.

15. Maier SE, West JR. Drinking patterns and alcohol-related birth defects. 
National Institute on Alcohol Abuse an Alcoholism (NIAAA) [Internet]. 2001 [cited 2003 Jul 25]. Available from: http://www.niaaa.nhl.gov/publications/ arh25-3/168-174.htm.

16. Usher R, McLean F. Intrauterine growth of live-born Caucasian infants at sea level: standards obtained from measurements in 7 dimensions of infants born between 25 and 44 weeks of gestation. J Pediatr.1969;74(6):901-10.

17. Sokol RJ, Martier SS, Ager JW. The TACE questions: practical prenatal detection of risk drinking. Am J Obstet Gynecol.1989;160(4):863-8.

18. Stoler JM, Holmes LB. Recognition of facial features of fetal alcohol syndrome in the newborn. Am J Med Genet. 2004;127(1):21-7.

19. Warren KR, Calhoun FJ, May PA, Viljoen DL, Li TK, Tanaka $\mathrm{H}$ et al. Fetal alcohol syndrome: an international perspective. Alcohol Clin Exp Res. 2001; 25(5 Suppl ISBRA):202S-6S.

20. Laranjeira R, Pinsky I, Zaleski. Caetano R. I Levantamento Nacional sobre os Padrões de Consumo de Álcool na População Brasileira. SENAD [Internet]. 2007 [acesso em 2 de abril de 2008]. Disponível em: http://www.senad.gov.br/ relatorio_padroes_consumo_alcool.pdf.
21. Passini Junior R. Alcohol consumption during pregnancy. Rev Bras Ginecol Obstet. 2005; 27(7):373-75.

22. May PA, Gossage P, Brooke LE, Hons BA, Snell CL, Marais AS et al. Maternal risk factors for fetal alcohol syndrome in the Western Cape Province of South Africa: a population-based study. Am J Public Health. 2005;95(7):1190-9.

23. Ethen MK, Ramadhari TA, Sheuerle AE, Canfield MA, Wyszynski DF, Druschel et al. Alcohol consumption by women before and during pregnancy [Internet]. 2008 [cited 2008 Aug 12]. Available from:

www.springerlink.com.w10155.dotlib.com.br/ content/qu5t630620726743/fultext.pdf.

24. Olegard R, Sabel KG, Aronsson M, Sandin B, Johansson PR, Carlsson C et al. Effects on the child of alcohol abuse during pregnancy. Retrospective and prospective studies. Acta Paediatr Scand Suppl. 1979; 68:112-21.

25. Freire TM, Machado JC, Melo EV, Melo DG. Efeitos do consumo de bebida alcoólica sobre o feto. Rev Bras Ginecol Obstet. 2005;27(7):376-81.

26. Atenção pré-natal. Manual puerpério [Internet]. 2006. [acesso em 20 de junho de 2008]. Disponível em: http:// www.saude.gov.br.

Recebido em:26/03/2008 Modificado em:12/08/2008 Aceito em:22/11/2008 